\title{
Dynamics of Atmospheric Regression Patterns: Regional Mountain Torque Events
}

\author{
JOSEPH EGGER \\ Meteorologisches Institut, Universität München, Munich, Germany \\ Klaus-Peter Hoinka \\ Institut für Physik der Atmosphäre DLR, Oberpfaffenhofen, Germany
}

(Manuscript received 30 June 2005, in final form 7 October 2005)

\begin{abstract}
The regression of atmospheric fields against a parameter $P$ with lag $\tau$ is a standard procedure in meteorology. Here, the torque exerted by a mountain massif is chosen as a parameter in order to study the interaction of weather systems with orography on a statistical basis. It is normally found that the amplitudes of the correlation patterns increase with $\tau \rightarrow 0$ and decrease for increasing positive lag. It is proposed to explain this ubiquitous feature in the orographic case on the basis of the covariance equations that govern these regressions. Two examples are discussed. First, a version of the low-order Charney-DeVore model of $\beta$-plane flow over a mountain is considered where stochastic forcing stirs a Rossby wave mode. It is found that the general increase of covariance amplitudes for $\tau \rightarrow 0$ (if it occurs) is mainly due to the forcing, but triple covariances of mountain torque and vorticity advection are important as well. A new covariance energy equation is derived to demonstrate that the frictional decay for $\tau>0$ is supported by these triple covariances while the stationary wave acts as a source for $\tau>0$. A dynamical interpretation of the triple terms is given. Next, data from the ECMWF 40-yr Re-Analysis (ERA-40) set are used to study mountain torque events in winter near Greenland, where the covariances of all standard variables with the torque $P$ exhibit a rapid quasi-barotropic increase with $\tau \rightarrow 0$ near Greenland. This amplification process is investigated by looking at the barotropic vorticity equation adapted to this statistical problem. This equation captures the evolution of the regression patterns reasonably well in the range $-2 \leq \tau \leq 2$ days. The triple covariances of torque and nonlinear vorticity advection play the key role in the amplification process. In particular, covariance enstrophy is generated and destroyed by these terms, a process without counterpart in the standard vorticity equation. Stochastic forcing is presumably unimportant. The interpretation of the triple terms is difficult in contrast to that of the other "linear" terms of the vorticity equation. The angular momentum in the Greenland domain decreases during events of positive torque.
\end{abstract}

\section{Introduction}

It is a standard method in meteorology to calculate lagged covariances and/or correlations of meteorological fields with parameters or with single station time series [see von Storch and Zwiers (1999) for a brief introduction to this technique]. For example, Wallace et al. (1988) correlate 500-hPa heights with the surface pressure at selected midlatitude stations for various lags. Wave patterns emerge that move eastward over the station with increasing lag $\tau$. The wave amplitude

Corresponding author address: Joseph Egger, Meteorologisches Institut der Universität München, Theresienstrasse 37, $80333 \mathrm{Mu}-$ nich, Germany.

E-mail: j.egger@lrz.uni-muenchen.de increases for $\tau \rightarrow 0$ but decreases for increasing positive lag. Wallace et al. (1988) estimate typical wave shapes, phase speeds, and even wave growths by comparing fields, say, for $\tau=-1$ day with those for $\tau=1$ day. Orographic effects on weather systems can be investigated by choosing the mountain torque as the key parameter. Weickmann (2003) correlates the mountain torque exerted by the Eurasian mountain massif with 200-hPa heights and surface pressure fields. Rossby waves are found to move over the plateau of Tibet during torque events while the surface pressure pattern appears to rotate anticyclonically near the mountain in addition to its eastward motion. Again, the amplitude of all covariance fields increases with $\tau \rightarrow 0$. Quite recently, Lott et al. (2004) related the Himalayan torque in the 20-30-day band to the 500-hPa height and found 
a rapid decay of the correlations between lag $\tau=0$ and the 3-day lag. Similar results with respect to Greenland will be presented below.

Whitaker and Sardeshmukh (1998, hereafter WS98) made a significant step toward the interpretation of synoptic eddy covariance fields such as presented by Wallace et al. (1988). They used a method introduced by Egger and Schilling (1983), where stochastically forced linear perturbations move in the observed mean flow. WS98 demonstrated that observed correlation maps for the Atlantic Ocean and Pacific Ocean storm track regions are reproduced quite well by such a model. The rapid growth of the eddy covariances for $\tau \rightarrow 0$ can be mainly attributed to energy transfer from the three-dimensional mean flow to the perturbations. The stochastic forcing is needed to maintain the eddy activity but the physical mechanisms behind the forcing are not specified by WS98. It is, however, not clear if the results of WS98 can be extended to the cases of mountain torque events just mentioned. For example, low-level flow splitting and blocking by mountains are truly nonlinear processes that elude linear modeling but may be important during torque events. Moreover, it is difficult to justify the incorporation of stochastic forcing if the resolved flow captures the relevant scales. Here, we turn directly to the equations that describe the evolution of the covariance fields with lag. These equations include the nonlinearities and need not take resort to stochastic forcing. Disadvantages of this approach will be revealed shortly.

Assume a standard nonlinear dynamical system

$$
\frac{d q_{i}}{d t}=a_{i j} q_{j}+b_{i j k} q_{j} q_{k}+f_{i},
$$

where $q_{i}(i=1, \ldots, I)$ are the variables, $a_{i j}$ and $b_{i j k}$ are constant coefficients, and $f_{i}$ forcing terms. Given the forcings and the constants, (1.1) can be integrated in time. We assume that there exists a statistically stationary ensemble of solutions to (1.1). The covariance function of an index $P(t)$ with $q_{i}$ is denoted by the symbol $C\left(P, q_{i} \mid \tau\right)$ where $q_{i}$ lags $P$. Assume, in particular, $\bar{P}=0$, where an overbar indicates the time mean and a prime indicates the deviation. Multiplying (1.1) by $P(t-\tau)$ and formation of expectations yields after simple manipulations

$$
\begin{aligned}
\frac{d}{d \tau} C\left(P, q_{i} \mid \tau\right)= & a_{i j} C\left(P, q_{j} \mid \tau\right)+b_{i j k} C\left[P,\left(q_{j} q_{k}\right) \mid \tau\right] \\
& +C\left(P, f_{i} \mid \tau\right) .
\end{aligned}
$$

These equations describe the evolution of the covariances of all variables with the index $P$ with lag. Unlike (1.1), the set (1.2) of equations cannot be integrated in time because there are no prognostic equations for the nonlinear terms $C\left[P,\left(q_{j} q_{k}\right) \mid \tau\right]$. These additional equations can be derived from (1.1) but, as is well known, this procedure leads to an infinite hierarchy of equations. The evaluation of the forcing terms may cause similar problems. What we can do, however, is to use data to evaluate the terms in (1.2) and to estimate their relative importance. Integration of (1.2) in time is possible if the nonlinear terms are prescribed according to observations.

In the following, the mountain torque exerted by a mountain massif on the axial component of the angular momentum of an atmospheric control volume will be chosen as the key parameter $P$. This torque characterizes the exchange of angular momentum between the solid earth and the atmosphere at the mountain. Its evolution in time is closely related to the synoptic development near the mountain (e.g., Weickmann 2003). Correspondingly, the role of mountain torques in local flow dynamics as well in the general atmospheric circulation has been discussed extensively in the past (e.g., Oort 1989; Bougeault et al. 1993; Czarnetzki 1997) both from an observational and a theoretical point of view. The examples quoted above show clearly that mountain torque events are suited for studying the amplification of covariances.

In addition to (1.2), it is useful to also write down the covariance version of a standard differential flow equation like the two-dimensional vorticity equation

$$
\frac{\partial}{\partial t} \zeta+\nabla_{2} \cdot[\mathbf{v}(\zeta+f)]=0
$$

( $\zeta$ is vorticity and $\mathbf{v}$ is horizontal velocity) to be used later. The $\beta$-plane approximation is accepted in (1.3) where the Coriolis parameter $f$ is represented by $f=$ $f_{0}+\beta y$. Using the same manipulations as above we arrive immediately at the statistical vorticity equation

$$
\begin{aligned}
\frac{\partial}{\partial \tau} C(P, \zeta \mid \tau) & +\nabla_{2} \cdot[\overline{\mathbf{v}} C(P, \zeta \mid \tau)]+\nabla_{2} \cdot[C(P, \mathbf{v} \mid \tau)(\bar{\zeta}+f)] \\
& +\nabla_{2} \cdot C\left(P, \mathbf{v}^{\prime} \zeta^{\prime} \mid \tau\right)=0 .
\end{aligned}
$$

The first term on the left-hand side describes the change of the covariance of the mountain torque and vorticity with lag. The second and third term on the left-hand side capture the interactions of perturbations with the time mean flow. The fourth term involves triple covariances and quantifies the advection of vorticity by the perturbation wind. A linear theory would include at least approximations to all terms except to the fourth one. Again, (1.4) cannot be integrated directly in time. 
We will first consider a low-order model of airflow over a mountain where data can be collected by numerical integration. The model is nonlinear but simple so that a fairly complete understanding of the growth and decay of the covariance fields is possible. Next we turn to observations to study the covariance of the mountain torque of Greenland with the pressure and flow fields near that massif.

\section{Covariance analysis of the Charney-DeVore (CDV) model}

\section{a. The model}

The low-order model of Charney and DeVore (1979) has been designed to explore the role of multiple equilibria in the atmosphere as generated by the interaction of zonal flow with topography. This model enjoyed some popularity over the years although its relevance to the atmosphere remained doubtful (Tung and Rosenthal 1985). Nevertheless, this model is still attractive (e.g., Crommelin and Majda 2004) because it contains important features of midlatitude large-scale dynamics. Here, we investigate a simplified threecomponent version, where stochastic forcing stirs a Rossby wave that interacts with the mean flow via the mountain torque (Egger 1981). The model domain is a zonally periodic $\beta$-plane channel. The model equations are

$$
\begin{aligned}
& \frac{d u_{0}}{d t}=-\delta u_{0}+a_{13} \psi_{i}+F_{1}, \\
& \frac{d \psi_{r}}{d t}=a_{23} \psi_{i}+k u_{0} \psi_{i}-d \psi_{r}+F_{2},
\end{aligned}
$$

and

$$
\frac{d \psi_{i}}{d t}=a_{31} u_{0}+a_{32} \psi_{r}-k u_{0} \psi_{r}-d \psi_{i}+F_{3},
$$

where

$$
\psi=\left[\psi_{r} \cos (k x)-\psi_{i} \sin (k x)\right] \sin (m y)
$$

is the streamfunction of the Rossby wave with real (imaginary) part $\psi_{r}\left(\psi_{i}\right) u_{0}(t)$ is the zonal mean flow and $h=h_{0} \cos (k x) \sin (m y)$ is the topography with zonal (meridional) wavenumber $k(\mathrm{~m})$. Damping has the time scales $d^{-1}$ and $\delta^{-1}$. The forcing term $F_{1}=\delta u^{x}$ is constant, while the white noise terms $F_{2}$ and $F_{3}$ represent the nonlinear interaction of the Rossby wave with unresolved smaller scales. The coefficients in (2.1)-(2.3) are $a_{13}=-k f_{0} h_{0} H^{-1}, a_{23}=\beta k K^{-2}, a_{31}=$ $k f_{0} h_{0} H^{-1} K^{-2}, a_{32}=-a_{23}$, with $K^{2}=k^{2}+m^{2}$ and $H$ as depth of the flow. The terms with the factors $a_{23}$ and $a_{32}$ encapsulate the Rossby wave dynamics, while the term $a_{31} u_{0}$ in (2.3) describes the orographic impact on the Rossby wave. Advection of the wave by the zonal wind is included as well in (2.2) and (2.3).

The parameter to be chosen is the mountain torque

$$
P=a_{13} \psi
$$

in (2.1). Actually $P$ would have to be multiplied by geometric factors to become a torque [see also (3.4)]. Note that $a_{13}$ is negative. This torque acts on the mean flow [see (2.1)], which is frictionally damped but also relaxed toward an imposed flow $u^{*}$. Kinetic energy is transferred from the mean flow to the wave if $u_{0} P<0$. The system performs a periodic orbit when damping and forcing are removed from (2.1)-(2.3) (Egger and Metz 1981). Energy and enstrophy are conserved in this case but the mean flow undergoes periodic oscillations, as does the wave energy, because of the orographically induced energy transfer.

\section{b. Results}

The parameters in the experiment to be discussed are chosen such that the equations support one equilibrium with $u_{0}=17 \mathrm{~m} \mathrm{~s}^{-1}\left[u^{*}=20 \mathrm{~m} \mathrm{~s}^{-1}, L=2 \pi / k=3 \times 10^{6}\right.$ $\mathrm{m}, h_{0}=1500 \mathrm{~m}, d=(2 \text { days })^{-1}$; and $\left.\delta=(4 \text { days })^{-1}\right]$. The width, $7 \times 10^{6} \mathrm{~m}$, of the channel is so large that the flow is essentially one-dimensional. The stationary waves induced by the mountain form an important part of the solution and exert a negative torque. The stochastic forcing is selected such that the standard deviation $\sigma_{u}=3.5 \mathrm{~m} \mathrm{~s}^{-1}$ of the zonal flow is relatively large, but the time mean value $\overline{u_{0}}=16.8 \mathrm{~m} \mathrm{~s}^{-1}$ is quite close to the model's equilibrium wind. Of course, many more parameter combinations have been considered, but we restrict our attention to this case where triple covariances are relatively important. In particular, a situation with multiple equilibria will not be investigated as being too far away from the Greenland case studied next.

Figure 1 shows the covariance $C(P, \psi \mid \tau)$ for $\tau= \pm 2$ days and $\tau=0$ days along the axis of the periodic channel. The mountain peak is located at $x=0$. To simplify the discussion we will assume a positive torque. Hence, the situation for $\tau=0$ reflects the basic configuration for $P>0$ with a high downstream $(x>0)$ of the mountain and a low upstream $(x<0)$. The evolution of the streamfunction pattern with lag is reminiscent of the findings of Weickmann (2003). The wave mode is moving eastward by nearly one wavelength in two days. Its phase velocity $c=15 \mathrm{~m} \mathrm{~s}^{-1}$ as estimated from Fig. 1 is in good agreement with the estimate $c=$ $13 \mathrm{~m} \mathrm{~s}^{-1}$ of the linear theory. The amplitude of the 


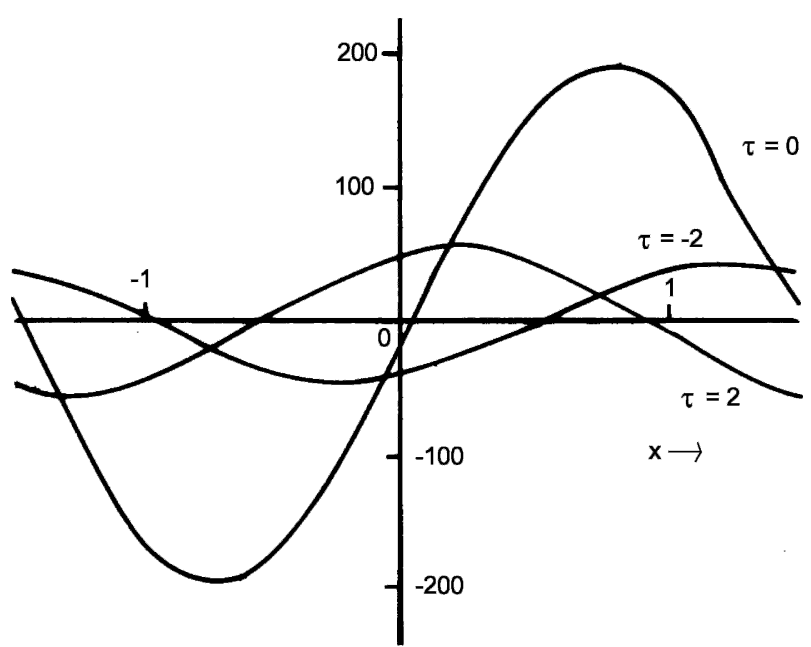

FIG. 1. Covariance $C(P, \psi \mid \tau)\left(\mathrm{m}^{3} \mathrm{~s}^{-3}\right)$ for $\tau= \pm 2$ days and $\tau=$ 0 days at the axis of the channel of zonal length $L=3 \times 10^{6} \mathrm{~m}$. The peak of the mountain is located at $x=0$ and distances are in $10^{6} \mathrm{~m}$.

covariance fields is quite small for $\tau=-2$ days when compared with that for $\tau=0$. The covariance $C\left(P, \psi_{I} \mid \tau\right)$ $=a_{13} C\left(\psi_{i}, \psi_{i} \mid \tau\right)$ as displayed in Fig. 2 (solid) is symmetric, of course, with respect to $\tau=0$. The reduction of the amplitude from the minimum at $\tau=0$ to the maxima at $\tau= \pm 1.3$ days is again conspicuous.

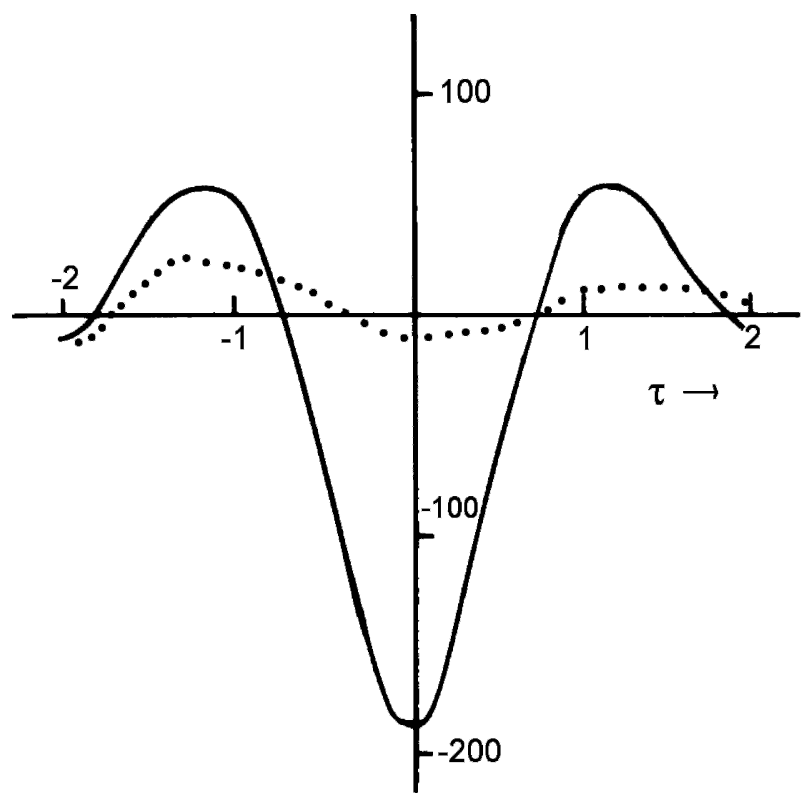

FIG. 2. Covariances $C\left(P, \psi_{i} \mid \tau\right)\left(\mathrm{m}^{3} \mathrm{~s}^{-3}\right)$ as a function of the lag $\tau$ (days) as obtained from the data and by integration of the covariance equations where the initial state at $\tau=-2$ days is specified according to the data. Both curves are identical at the level of drawing accuracy. The dotted curve gives the results of the integration when the forcing terms and the turbulence terms in (2.7) and (2.8) are suppressed.
Let us turn to the model's covariance equations corresponding with (1.2)

$$
\begin{aligned}
\frac{d}{d \tau} C\left(P, u_{0} \mid \tau\right)= & -\delta C\left(P, u_{0} \mid \tau\right)+a_{13} C\left(P, \psi_{i} \mid \tau\right), \\
\frac{d}{d \tau} C\left(P, \psi_{r} \mid \tau\right)= & -d C\left(P, \psi_{r} \mid \tau\right)+a_{23} C\left(P, \psi_{i} \mid \tau\right) \\
& +k\left(\bar{u}_{0} C\left(P, \psi_{i} \mid \tau\right)+\bar{\psi}_{i} C\left(P, u_{0} \mid \tau\right)\right. \\
& +C\left[P,\left(u_{0}^{\prime} \psi_{i}^{\prime}\right) \mid \tau\right]+C\left(P, F_{2} \mid \tau\right),
\end{aligned}
$$

and

$$
\begin{aligned}
\frac{d}{d \tau} C\left(P, \psi_{i} \mid \tau\right)= & a_{31} C\left(P, u_{0} \mid \tau\right)+a_{32} C\left(P, \psi_{r} \mid \tau\right) \\
& -d C\left(P, \psi_{i} \mid \tau\right)-k\left(\bar{u}_{0} C\left(P, \psi_{r} \mid \tau\right)\right. \\
& +\bar{\psi}_{r} C\left(P, u_{0} \mid \tau\right)+C\left[P,\left(u_{0}^{\prime} \psi_{r}^{\prime}\right) \mid \tau\right] \\
& +C\left(P, F_{3} \mid \tau\right) .
\end{aligned}
$$

The stationary wave is represented explicitly in (2.7) and (2.8). The triple covariances describe the covariance of $P$ and vorticity advection by the anomalous zonal wind. These equations are satisfied quite well by the covariances derived from a run of about $22 \mathrm{yr}$. This is demonstrated in Fig. 2 where the results of a direct integration of (2.6)-(2.8) are displayed with initial state at $\tau=-2$ days specified according to observations. Forcing and triple covariances are also specified as functions of lag. The difference between the observations and numerical results is below drawing accuracy. To demonstrate the role of forcing and triple terms, the integration is repeated but with these terms omitted. The resulting covariance $C\left(P, \psi_{i} \mid \tau\right)$ (Fig. 2; dotted) represents the frictional decay of the Rossby wave. A repetition of the integration with the triple covariances included results in enhanced amplitudes (not shown) but stochastic forcing is clearly dominant. Omission of the stationary wave terms leads to oscillations that differ substantially from those in Fig. 2. That is not surprising, because the stationary wave forms an important part of the model climate.

Further information on the role of the triple covariances is obtained from the energetics of (2.6)-(2.8) after introducing the covariance energy

$$
\begin{aligned}
\mathrm{CE}(\tau)= & \frac{1}{2}\left\{C\left(P, u_{0} \mid \tau\right)^{2}+K^{2}\left[C\left(P, \psi_{r} \mid \tau\right)^{2}\right.\right. \\
& \left.\left.+C\left(P, \psi_{i} \mid \tau\right)^{2}\right]\right\} .
\end{aligned}
$$


It follows

$$
\begin{aligned}
\frac{d}{d \tau} \operatorname{CE}(\tau)= & -2 d \operatorname{CE}(\tau)+k K^{2} C\left(P, u_{0} \mid \tau\right)\left[\bar{\psi}_{i} C\left(P, \psi_{r} \mid \tau\right)\right. \\
& \left.-\bar{\psi}_{r} C\left(P, \psi_{i} \mid \tau\right)\right] \\
& +k K^{2}\left\{C\left(P, \psi_{r} \mid \tau\right) C\left[P,\left(u_{0}^{\prime} \psi_{i}^{\prime}\right) \mid \tau\right]\right. \\
& \left.-C\left(P, \psi_{i} \mid \tau\right) C\left[P,\left(u_{0}^{\prime} \psi_{r}^{\prime}\right) \mid \tau\right]\right\} \\
& +K^{2}\left[C\left(P, \psi_{r} \mid \tau\right) C\left(P, F_{2} \mid \tau\right)\right. \\
& \left.+C\left(P, \psi_{i} \mid \tau\right) C\left(P, F_{3} \mid \tau\right)\right]
\end{aligned}
$$

The first term on the right-hand side of (2.10) describes the effect of damping where we assume $\delta=d$ for the sake of simplicity. The second term represents the interaction of the flow with the stationary wave and the last term the impact of forcing. Corresponding terms are found as well in standard energetics of (2.1)-(2.3) for the eddy energy in the climatic mean state. What is new is the third term that contains the triple correlations. It is displayed in Fig. 3 as a function of lag (solid) together with the dissipation term (dotted) and the standing wave term (dashed). The dissipation is essentially opposite to the covariance energy and indicates a dramatic increase of covariance energy from $\tau=-1$ day until $\tau=\sim 0$. There is a slight asymmetry with respect to $\tau=0$ and a rapid decline for $\tau>6 \mathrm{~h}$. The stationary wave provides a fairly large energy input for $-0.3 \leq \tau \leq 0.8$ days. The main contribution to the stationary wave term comes from the high $\overline{\psi_{r}}>0$ above the mountain. Assume a situation where $P>0\left(\psi_{I}<0\right)$, so that the anomaly of $u_{0}$ is growing and becomes positive for $\tau \geq 0$. These anomalous westerlies advect the anticyclonic vorticity of the stationary wave into the lee where, therefore, $\psi_{I}$ decreases further so that an overall increase of energy is caused by this effect. This argument is similar to that proposed by Jin and Ghil (1990) to explain topographic instability. The model's equilibrium is, however, stable and we witness in Fig. 3 an energy transfer from the stationary wave that is also underlying topographic instability. The stochastic forcing term increases exponentially (not shown) from $\tau=$ -4 days until $\tau=0$ to reach a maximum of $13.6 \times 10^{-13}$ $\mathrm{m}^{6} \mathrm{~s}^{-6}$. This term vanishes for $\tau>0$ because white noise does not have a memory. Hence, the sum of all curves for $\tau>0$ represents the right-hand side of (2.10). Figure 3 demonstrates clearly that the triple covariances contribute substantially to the growth for $\tau<0$ and to the decay for $\tau>0$.

A closer inspection of the triple correlations shows that the first term in the brackets in (2.10) is the more important one in this case. The factor $C\left(P, \psi_{r} \mid \tau\right)$ is an-

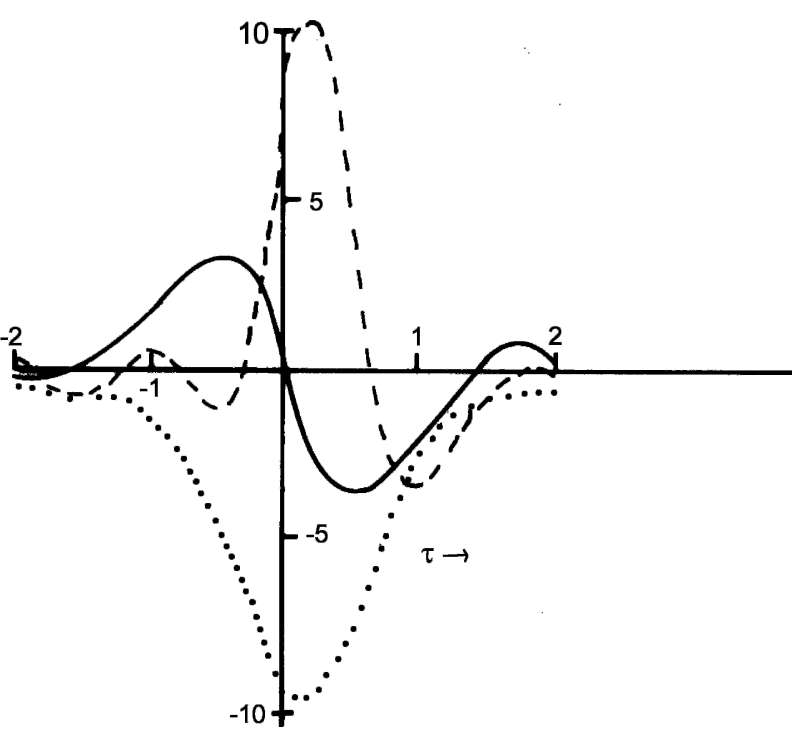

FIG. 3. Contributions of the triple correlations (bold), standing wave field (dashed), and dissipation (dotted) to the rhs of (2.10) $\left(10^{-13} \mathrm{~m}^{6} \mathrm{~s}^{-6}\right)$ as a function of lag $\tau$ (days).

tisymmetric with respect to $\tau=0$, because $\psi_{r} \sim 0$ for a strong torque event. Moreover, this factor is positive for $\tau<0$ because the wave propagates eastward and the high located in the lee at $\tau=0$ must have been above the mountain for $\tau \rightarrow 0$. The triple covariance $C(P$, $\left.u_{0}^{\prime} \psi_{i}^{\prime} \mid \tau\right)$ itself is proportional to $-C\left(\psi_{i}^{\prime 2}, u_{0}^{\prime} \mid 0\right)$ near $\tau=$ 0 where it has to be positive. Assume, for example, that $u_{0}^{\prime}>0$ near $\tau=0$ so that the kinetic energy of the mean flow is relatively large. Hence, the wave energy and thus $\psi_{i}^{\prime 2}$ must be below its mean value. If $u_{0}^{\prime}<0$, it is the reverse and, therefore $-C\left(\psi_{i}^{\prime 2}, u_{0}^{\prime} \mid 0\right)>0$. The evaluation of this term shows that the sign of $C\left(P, u_{0}^{\prime} \psi_{i}^{\prime} \mid \tau\right)$ does not change for $|\tau|<2$ days. The combination with the antisymmetric factor $C\left(P, \psi_{r} \mid \tau\right)$ gives the main contribution to the triple term curve in Fig. 3. Note that neither damping nor forcing enter this argumentation. We speculate, therefore, that at least the shape of the triple terms can be understood by looking at the periodic orbits where the above arguments are valid as well.

Altogether it follows that the intensification of the streamfunction covariance for $\tau \rightarrow 0$ in Fig. 1 is mainly due to the stochastic forcing, but triple covariances play an important role as well. The decay is caused by friction and by the triple covariances, but is opposed by the impact of the stationary wave. As compared with WS98, we observe a dominance of stochastic forcing, energy transfer from the time mean flow only in the decay phase, and, as a completely new feature, support for covariance growth and decay by the triple terms. 


\section{Airflow over and around Greenland: Observations}

The wealth of flow phenomena observed near the huge meridionally oriented barrier of Greenland has attracted both synopticians and theoreticians for some time. Cyclones moving toward Greenland from the west tend to be split (Walden 1959; Putnins 1970). The low-level disturbance moves northward along the west coast while the other part crosses the barrier or may go around the southern tip of Greenland. Jets are observed to form downstream of the southern tip of Greenland (Doyle and Shapiro 1999). Scorer (1988) suggested that the Icelandic low is essentially a leeside low that owes its existence to the Greenland barrier (see, however, Kristjánsson and McInnes 1999). Maps of cyclone activity (Hoskins and Hodges 2002) provide further information on the dynamic climatology. The interaction of cyclones with Greenland must be reflected in variations of the mountain torque as is also true for the growth and decay of blocks above the Atlantic [see Schwierz (2001) for a detailed case study]. All in all, Greenland is embedded in a dynamically active region and it is reasonable to expect that covariance fields will undergo rapid changes with lag if the Greenland torque is chosen as data stratification parameter. In what follows, we pursue the same strategy as above but now have atmospheric observations at our disposal.

The data to be used are part of the European Centre for Medium-Range Weather Forecasts (ECMWF) 40yr Re-Analysis (ERA-40) dataset covering the period January 1958-December 2001 (Simmons and Gibson 2000). The domain $49.5^{\circ}-81^{\circ} \mathrm{N}, 81^{\circ} \mathrm{W}-0^{\circ}$ is selected for the analysis. All data are transformed such that they are available at the points of a $1.125^{\circ} \times 1.125^{\circ}$ horizontal grid and at 13 constant height surfaces with a vertical spacing $D z=1000 \mathrm{~m}$ with the lowest level at $z=500 \mathrm{~m}$.

The mountain torque $P$ for the Greenland massif is calculated on the basis of the available surface pressure fields and the topographic profile of the reanalysis project. It results from an integration of the axial angular momentum equation

$$
\frac{\partial}{\partial t} m_{3}+\nabla \cdot\left(\mathbf{v} m_{3}\right)=-\frac{\partial p}{\partial \lambda}
$$

over the analysis volume $V\left(81^{\circ} \mathrm{W}=\lambda_{1}<\lambda<\lambda_{2}=0^{\circ}\right.$, $\varphi_{1}=9.5^{\circ} \mathrm{N}<\varphi<\varphi_{2}=81^{\circ} \mathrm{N}, h<z \leq 13 \mathrm{~km}$; where $\lambda$ is longitude, $\varphi$ is latitude, $h$ is topography, and $p$ is pressure) covering the obstacle. In (3.1),

$$
m_{3}=\rho(u+\Omega a \cos \varphi) a \cos \varphi
$$

is the axial angular momentum per unit volume ( $\rho$ is density, $a$ is the earth's radius, and $\Omega=2 \pi$ day $^{-1}$ ). The total angular momentum $M_{3} \int_{V}=m_{3} d V$ is affected via

$$
\begin{aligned}
\frac{d M_{3}}{d t}= & - \text { boundary fluxes }-\int_{\varphi_{1}}^{\varphi_{2}} \int_{h}^{\infty}\left[p\left(\lambda_{2}, \varphi, z\right)\right. \\
& \left.-p\left(\lambda_{1}, \varphi, z\right)\right] a d z d \varphi+P+T_{f}
\end{aligned}
$$

by fluxes of angular momentum through the lateral boundaries, by a pressure torque, and by the mountain torque

$$
P=-\int_{F} p_{s} \frac{\partial h}{\partial \lambda} d F,
$$

where $p_{s}$ is the surface pressure and by the friction torque $T_{f}$. We wish to separate the mountain torque $P$ of Greenland from that exerted by other topographic features west of this island by choosing the domain $F$ of integration in (3.4) properly. The zonal boundaries of the area $F$ are defined by the first zonal grid points to the east and west of Greenland where the surface height drops below zero. These heights are set to zero and the actual surface pressure at these points is reduced to this level. This allows us to calculate the mountain torque exerted exclusively by Greenland. The torque is positive if the surface pressure to the east of the crest line of Greenland is larger than in the west. Note that the area $F$ is used only for the evaluation of the mountain torque.

The covariance functions of pressure, horizontal wind, temperature, potential temperature, vorticity, and divergence with $P$ are evaluated per day for every season at every grid point for all layers up to a height of $12500 \mathrm{~m}$. Moreover, the triple terms $C[P,(\mathbf{v} \zeta) \mid \tau]$ are computed as well.

\section{Results}

We present only the results for the winter season (December-February) where the impact of Greenland is particularly pronounced.

\section{a. Mean fields}

Although a detailed discussion of the wintertime mean state is beyond the scope of this paper, a brief presentation is warranted. The horizontal mean flow $\overline{\mathbf{v}}$ at $z=5500 \mathrm{~m}$ is displayed in Fig. 4a. Its structure reflects the wintertime trough above Canada with southerlies above Greenland. The northern flank of the jet stream is seen in the southern part of the domain. Higher up $(z=\sim 10 \mathrm{~km})$ the flow is more intense but 


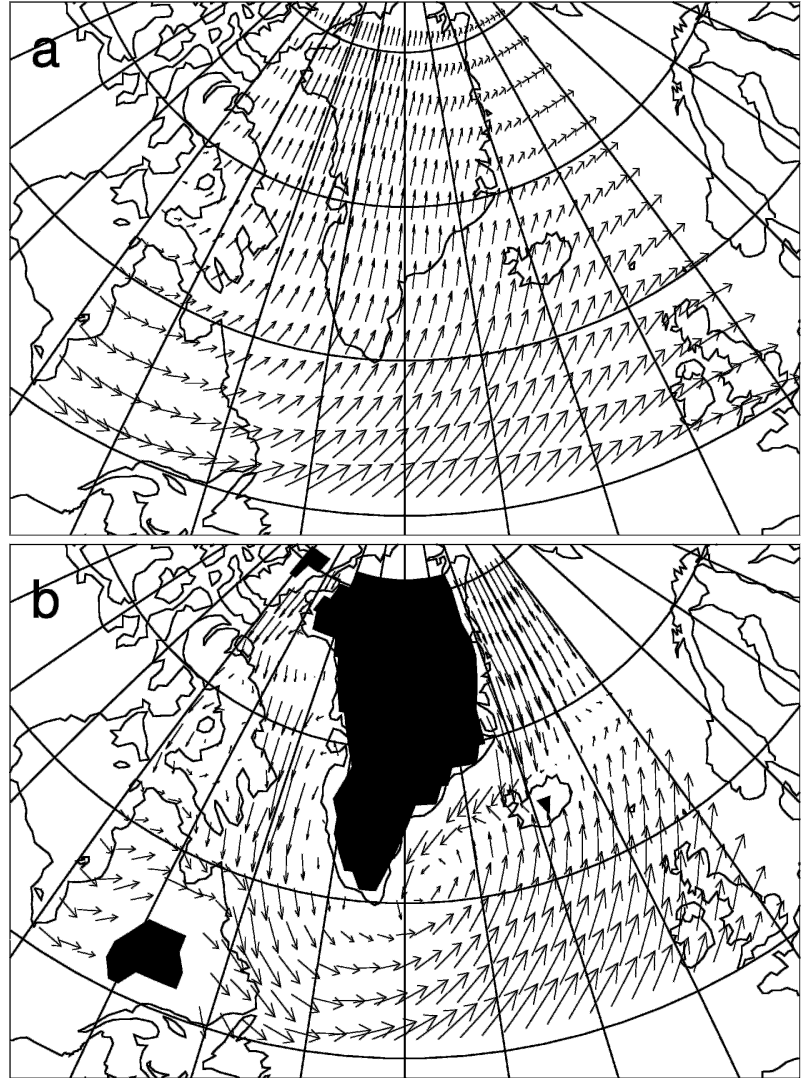

FIG. 4. Mean horizontal winds at levels (a) $z=5500 \mathrm{~m}$ (maximum flow velocity $20.4 \mathrm{~m} \mathrm{~s}^{-1}$ ) and (b) $z=500 \mathrm{~m}$ (maximum flow velocity $8.0 \mathrm{~m} \mathrm{~s}^{-1}$ ). The dark black color indicates topography.

otherwise quite similar to that in Fig. 4a. The most prominent feature at the surface (Fig. $4 \mathrm{~b}$ ) is the cyclonic flow of the Icelandic low with its northwestern branch constrained by Greenland. The layer of southward flow west of Greenland is fairly shallow and is replaced by southerlies at the next levels. We are not aware of any attempts to explore the linear stability characteristics of this mean flow in the presence of this huge obstacle. It is clear, however, that there are substantial stationary waves in the research domain similar to the situation in the CDV model and that dealt with by WS98.

\section{b. Covariance fields}

The autocovariance $P$ of the mountain torque decays rapidly with lag (Fig. 5) with a variance of $46 \mathrm{Ha}^{2}$ [where 1 Hadley $\left.(\mathrm{Ha})=10^{18} \mathrm{~J}\right)$. Strong torque events have a lifetime of 3-4 days. There is, however, a tail with slow decay. If a torque of $1 \mathrm{Ha}$ would act on the mass of the atmosphere in the analysis domain for 8 days and if this torque would just accelerate the zonal velocity, an eastward mean velocity of $\sim 1 \mathrm{~m} \mathrm{~s}^{-1}$ would be induced. In other words, a typical torque event of a

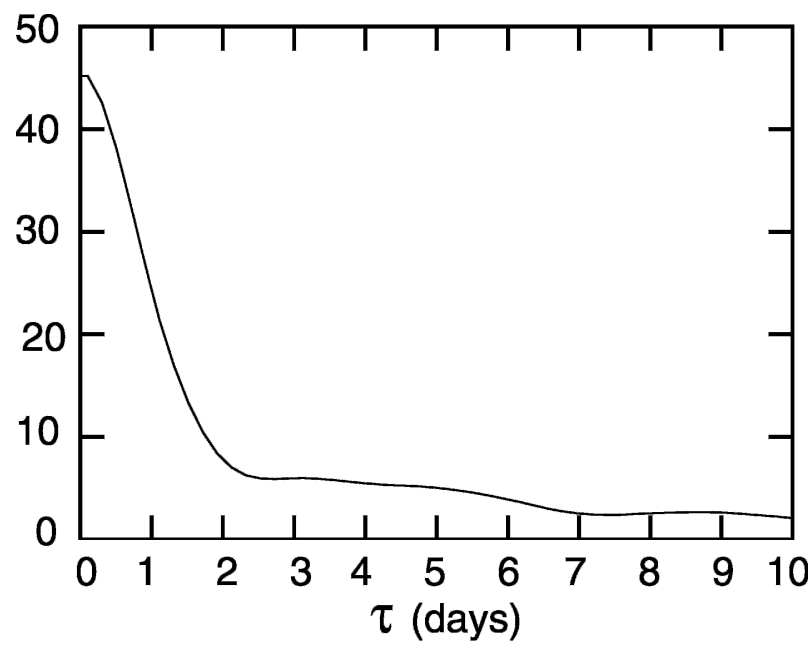

Fig. 5. Autocovariance of the Greenland mountain torque $P$ $\left(\mathrm{Ha}^{2}\right)$ as a function of lag $\tau$ (days).

mean amplitude of approximately $5 \mathrm{Ha}$ and a duration of 4 days could induce a velocity increase of $2.5 \mathrm{~m} \mathrm{~s}^{-1}$. The autocovariances of $P$ in other seasons are fairly similar in shape to that in Fig. 5, but the variances are less with $\sim 30 \mathrm{Ha}^{2}$ in boreal spring and fall and $12 \mathrm{Ha}^{2}$ in boreal summer. The winter mean torque is $2.2 \mathrm{Ha}$ (annual mean $1.4 \mathrm{Ha}$ ).

In the following presentation of covariances, the torque $P$ is normalized by the wintertime standard deviation $\sigma_{p}=6.7 \mathrm{Ha}$. To facilitate the discussion of covariance fields we will assume $P>0$ in what follows. The normalized covariance fields of $P$ and the pressure at $500 \mathrm{~m}$ are displayed in Fig. 6. The near surface pressure fields for $\tau<-1$ day exhibit little distinct features. The pressure tends to be higher in the northern part of the analysis domain. As an example, we show in Fig. 6a the pressure at $z=500 \mathrm{~m}$ for $\tau=-2$ days. The pressure anomaly is higher to the east of Greenland than in the west with a closed 4-hPa isobar northwest of Iceland. This means that a positive torque is exerted at that time (see also Fig. 5). There is a low above Quebec. This pressure distribution implies anomalous easterly flow in most parts of the domain. At $z=5500 \mathrm{~m}$, there is a high above Greenland that is almost symmetric with respect to the axis of this massif (Fig. 6b). The corresponding flow is anticyclonic and predominantly easterly (not shown). The flow anomalies related to the torque events extend throughout the troposphere. There is a weak anticyclonic flow structure above Greenland even at $z=11500 \mathrm{~m}$ (not shown).

The near-surface high in the east intensifies during the following day and attains an amplitude of $6 \mathrm{hPa}$ at $\tau=-1$ day (Fig. 6c). Moreover, the central pressure of the low in the southwest is now $\sim-6 \mathrm{hPa}$ and its posi- 


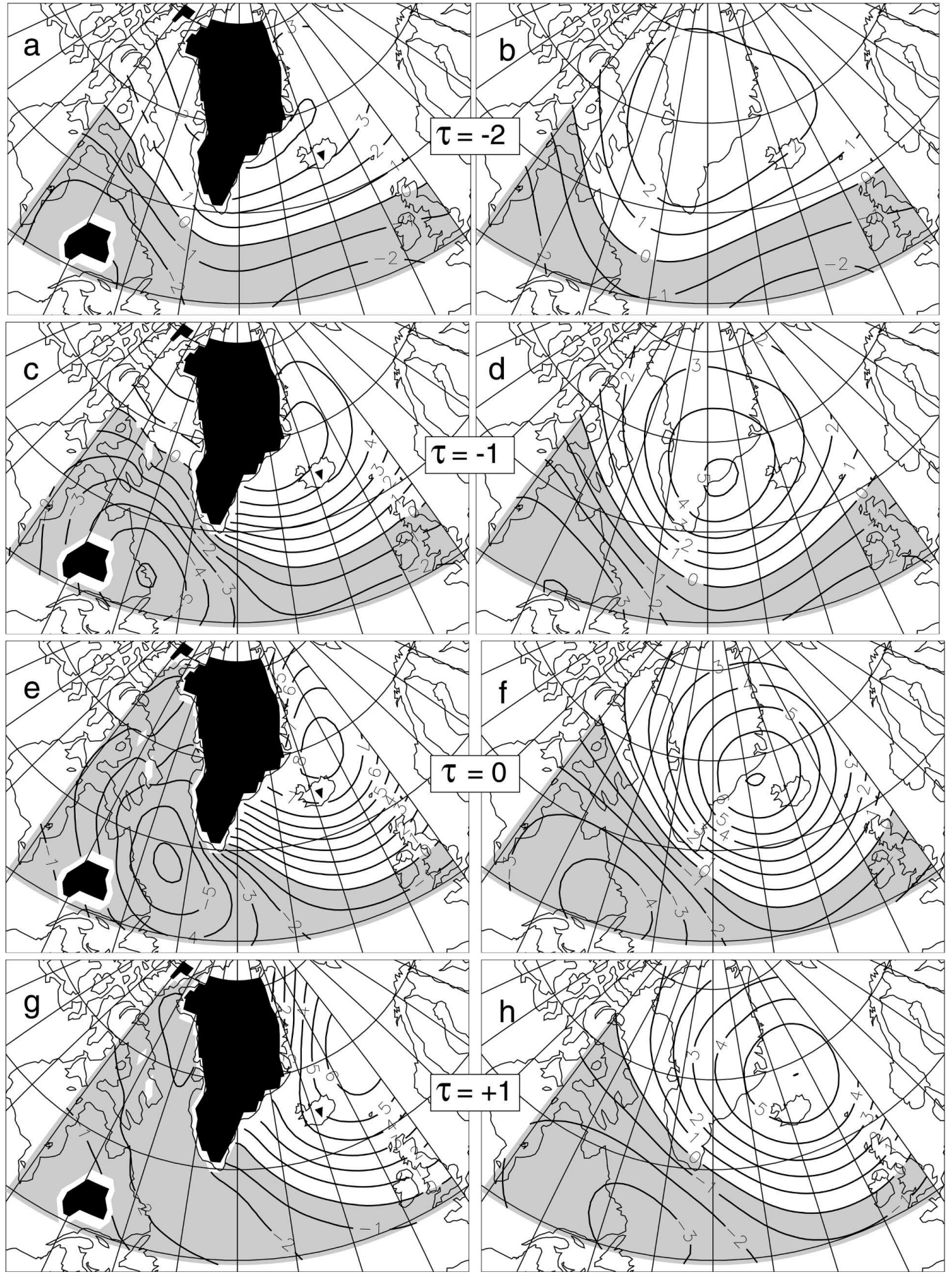

FIG. 6. Crosscovariance function $C(P, p \mid \tau)$ of the Greenland mountain torque at (left) $z=500 \mathrm{~m}$ and (right) $z$ $=5500 \mathrm{~m}$ with the pressure field normalized by the standard deviation $\sigma_{p}$ of $P$ so that the resulting fields can be interpreted in $\mathrm{hPa}$.

tion is shifted slightly to the east. The high at $5500 \mathrm{~m}$ is intensifying as well and begins to move eastward (Fig. $6 \mathrm{~d}$ ). At $\tau=0$ (Fig. 6e), the pressure anomaly at $z=500$ $\mathrm{m}$ is negative everywhere to the west of Greenland with a minimum above the Labrador Sea. The high in the east now has a central pressure of $\sim 8.0 \mathrm{hPa}$ and is located to the north of Iceland. The pressure gradients are largest in the southern part of Greenland. The east- 


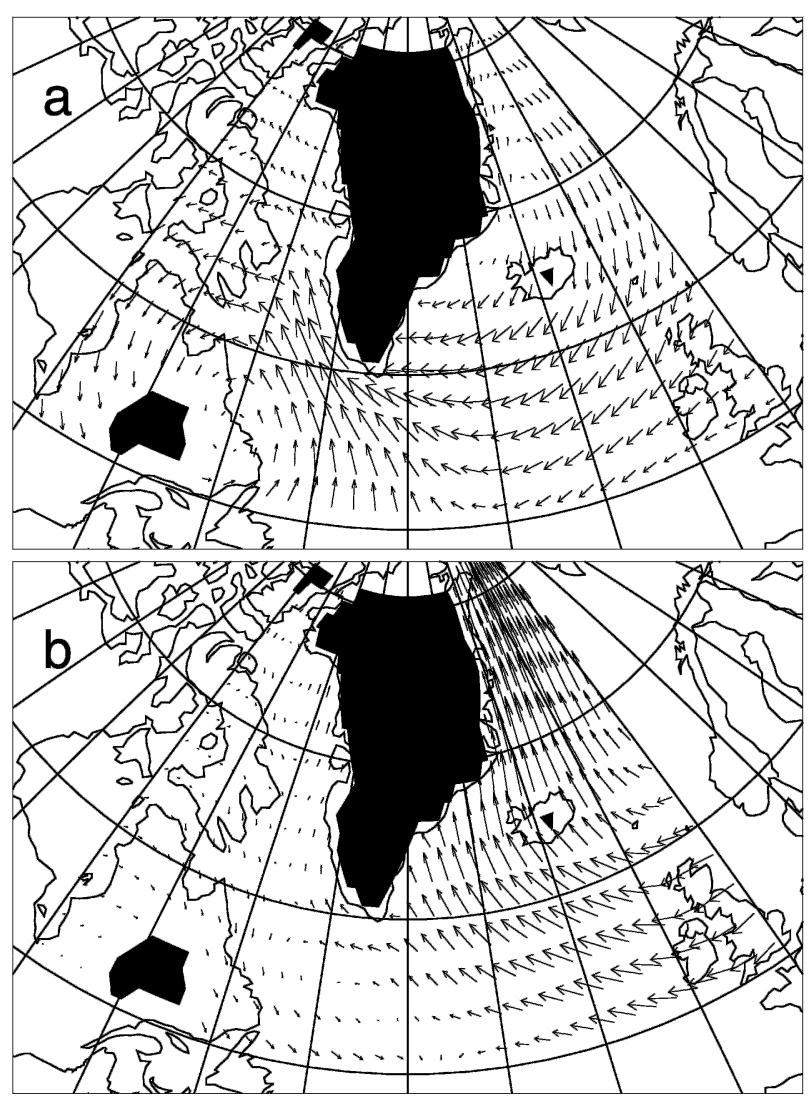

FIG. 7. Crosscovariance $C(P, \mathbf{v} \mid \tau)$ of the normalized Greenland mountain torque with the horizontal wind at $z=500 \mathrm{~m}$ at (a) $\tau=$ -1 day ( $m a x$ velocity $7.2 \mathrm{~m} \mathrm{~s}^{-1}$ ) and (b) $\tau=2$ days ( $\max$ velocity $\left.3.2 \mathrm{~m} \mathrm{~s}^{-1}\right)$.

ward motion of the pressure patterns is seen higher up as well. The center of the high at $z=5500 \mathrm{~m}$ is found above the east coast of Greenland. The low in the south has closed isobars as well (Fig. 6f). The pressure increase in the high's center is $5 \mathrm{hPa}$ over the last two days. There is even a weak anticyclonic signature of the event in the stratosphere (not shown). One day later, the surface anticyclone is about to leave the analysis domain (Fig. $6 \mathrm{~g}$ ). The low in the south disappeared. At $z=5500 \mathrm{~m}$, both centers are still within the budget area so that we find a westward tilt of the axes with height (Fig. 6h). Eastward motion and decay of the amplitudes continues with increasing $\tau$. The pressure fields at $\tau=5$ days are fairly similar to those at $\tau=-5$ days.

By and large, the regressed winds are geostrophic as one would expect. The near-surface wind is strongest at $\tau=-1$ day with a maximum velocity of $7.2 \mathrm{~m} \mathrm{~s}^{-1}$ near the tip of Greenland (Fig. 7a). This flow around the tip becomes quite weak for $\tau>1$ day when the high's anticyclonic circulation moves eastward (Fig. 7b). Vertical motion is strongest above the southern part of

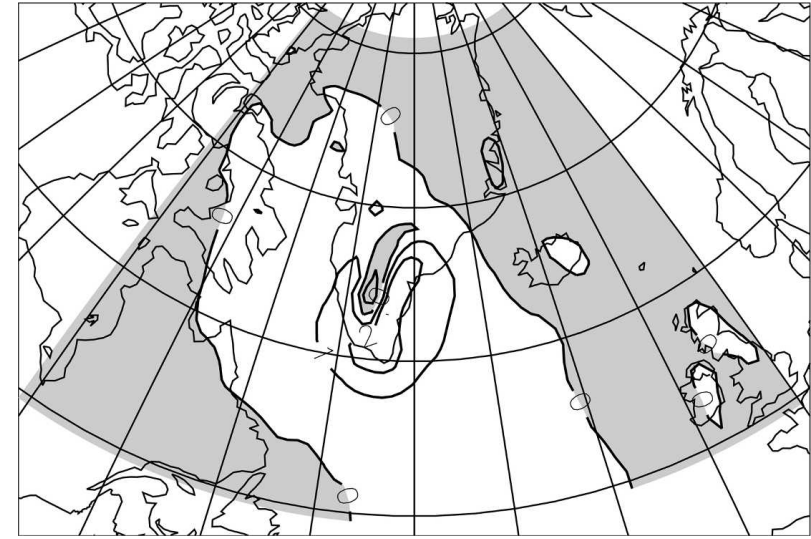

FIG. 8. Crosscovariance $C(P, w \mid \tau)$ of the normalized mountain torque with the vertical velocity $\left(\mathrm{cm} \mathrm{s}^{-1}\right)$ at $\tau=0$ days and $z=$ $3500 \mathrm{~m}$.

Greenland (Fig. 8). The strong southeasterly flows there induce upward motion above the east coast and sinking above the crest. Maximum velocities are as large as $3 \mathrm{~cm} \mathrm{~s}^{-1}$.

The evolution of the covariance enstrophy

$$
Z=\frac{1}{2 N} \sum_{i} C(P, \zeta \mid \tau)^{2} \cos \varphi_{i}
$$

( $i$ is a grid point index and $N$ is the number of grid points) provides a convenient measure of the growth and decay of the covariance fields. There is a rapid increase from $\tau=\sim-2$ days until $\tau=\sim 0$ with a slight asymmetry so that the maximum occurs for positive lags (Fig. 9). The decay of the covariance fields is as

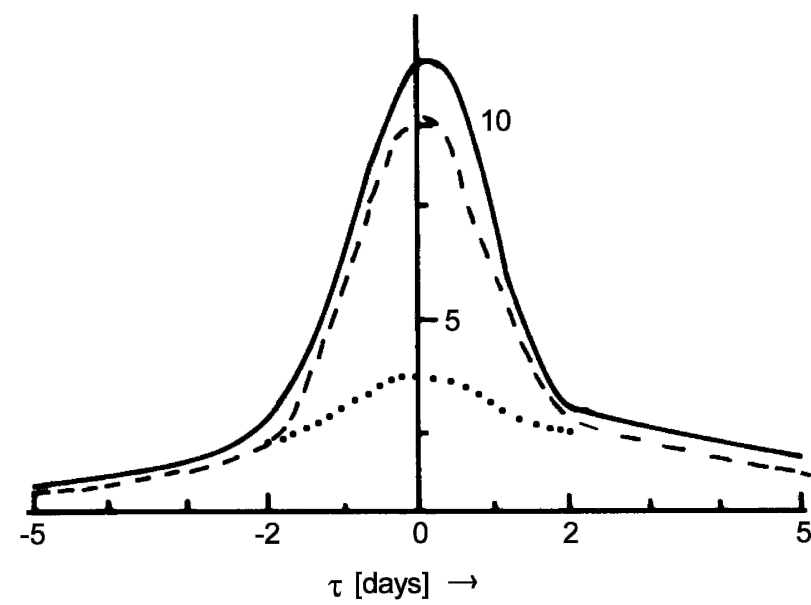

FIG. 9. Covariance enstrophy $\left(10^{-12} \mathrm{~s}^{-2}\right)(4.1)$ as a function of lag (bold) as observed, enstrophy of the vertical mean vorticity (dashed), and the corresponding enstrophy of the integration of the linear vorticity equation (dotted) from $\tau=-2$ days to $\tau=+2$ days. 
rapid as the increase. Also given is the enstrophy of the vertical mean vorticity (dashed), which is rather close to the enstrophy itself. Clearly, the flow evolution is quasibarotropic.

One may expect on the basis of (3.3) that the angular momentum contained in the analysis domain increases for $\tau \rightarrow 0$ just as in the CDV model because the mountain torque acts as a source. It is found, however, that the wind term

$$
M_{w}=\int_{v} \bar{\rho} C(P, u \mid \tau) a \cos \varphi d V
$$

decreases from $-34 \times 10^{22} \mathrm{~J}$ s at $\tau=-5$ days to a minimum of $-82 \times 10^{22} \mathrm{~J}$ s at $\tau=0$ to increase later on. This decrease of $M_{w}$ for $\tau \rightarrow 0$ reflects the strengthening of the easterlies in the flow domain for $\tau \rightarrow 0$. The mass term

$$
M_{m}=g^{-1} \int_{s} C\left(P, p_{s} \mid \tau\right) \Omega(a \cos \varphi)^{2} d S
$$

is just $\sim 10 \%$ of the wind term. It increases first for $\tau \rightarrow$ 0 , but decreases when the low pressure systems enter the domain (see Fig. 6). The torque provides an input of $\sim 80 \times 10^{22} \mathrm{~J}$ s within the interval $-5 \leq \tau \leq 0$ days. The pressure torque turns out to be negligible so that the boundary fluxes dominate. We have to conclude that a positive torque event does not lead to an increase of the zonal flow in the analysis domain. In contrast, Czarnetzki (1997) found a decrease of the regional angular momentum for cases of Rocky Mountain lee cyclogenesis when a negative mountain torque is exerted. However, the boundary fluxes and the pressure torque were by far the largest terms.

\section{c. Vorticity equation}

In principle, the analysis of the Greenland case could proceed the same way as for the CDV model, that is, we could write down and evaluate all terms of the covariance equations (1.2) governing the flow near Greenland. This is a task of considerable complexity that will not be tackled. Instead, we rely on (1.3) and (1.4) to shed some light on the role of various processes. It is clear that (1.3) involves many assumptions. In particular, vertical advection effects are excluded as are the solenoidal term and the impact of friction. Nevertheless, it is reasonable to believe that (1.3) and (1.4) capture basic aspects of the dynamics of the flow over and around Greenland. It appears appropriate to analyze (1.4) in vertically integrated form so that

$$
T=J^{-1} \sum_{j} \frac{\partial}{\partial \tau} C(P, \zeta \mid \tau)
$$

is the mean tendency where the index $j$ runs over all layers above the topography and $J=13$ is the total number of layers. The total covariance of the flux of relative vorticity

$$
\gamma=J^{-1} \sum_{j} C(P, \mathbf{v} \zeta \mid \tau)
$$

is composed of

$$
\begin{aligned}
& \gamma_{1}=J^{-1} \sum_{j} C(P, \zeta \mathbf{v} \mid \tau), \\
& \gamma_{2}=J^{-1} \sum_{j} C(P, \mathbf{v} \bar{\zeta} \mid \tau), \quad \text { and } \\
& \gamma_{3}=J^{-1} \sum_{j} C\left(P, \mathbf{v}^{\prime} \zeta^{\prime} \mid \tau\right) .
\end{aligned}
$$

The contribution by the linear terms to (4.4) is

$$
D_{l}=-\nabla_{2} \cdot\left(\gamma_{1}+\gamma_{2}\right) \text {. }
$$

The triple covariances in (1.4) represent turbulent fluxes with divergence

$$
D_{t}=-\nabla_{2} \cdot \gamma_{3}
$$

Finally, there is the vertically integrated divergence term

$$
D_{0}=-f_{0} J^{-1} \sum_{j} C(P, \boldsymbol{\nabla} \cdot \mathbf{v} \mid \tau) \sim \frac{f_{0} J^{-1} C\left(P, \mathbf{v}_{s} \cdot \boldsymbol{\nabla} h \mid t\right)}{D z},
$$

which should be close to the orographic vorticity generation term $\left(\mathbf{v}_{s}\right.$ is the horizontal surface wind and $f+$ $\bar{\zeta}$ is replaced by $f_{0}$ ) and the $\beta$ term

$$
B=-J^{-1} \sum_{j} \beta C(P, v \mid \tau) .
$$

Altogether, we wish to test the expression

$$
T=D_{l}+D_{t}+D_{0}+B
$$

by comparing the observed tendency $T$ to the righthand side $R$ as observed. In principle, a stochastic forcing term could have been added to (1.4), but there is no reason to believe that the impact of subscale motions is important given the good resolution of the data.

A gross measure of the quality of (4.11) as a forecast equation for the situation at hand is obtained by evaluating pattern correlation coefficients $K(T, R)$ for all tendencies at the grid points of the analysis domain and various combinations $R$ of terms on the right of (4.11) (Table 1). The tendencies are evaluated as centered 
TABLE 1. Pattern correlation coefficients $K$ (first entry per column) of the tendency $T$ at all grid points of the analysis domain with various combinations of terms on the rhs of (4.11) for various lags. Also given (second entry per column) is the ratio $\sigma_{T} / \sigma_{R}$ of the standard deviation $\sigma_{T}$ and of the rhs $\sigma_{R}$ of the test equation.

\begin{tabular}{cccc}
\hline \hline $\begin{array}{c}\text { Lag } \\
\text { (days) }\end{array}$ & $\begin{array}{c}K\left(T, D_{t}+\right. \\
\left.D_{l}+B, \tau\right)\end{array}$ & $\begin{array}{c}K\left(T, D_{t}+D_{l}+\right. \\
B+D, \tau)\end{array}$ & $K\left(T, D_{t}, \tau\right)$ \\
\hline-4 & $0.19 / 0.2$ & $0.19 / 0.2$ & $0.03 / 1.0$ \\
-3 & $0.31 / 0.3$ & $0.28 / 0.3$ & $0.21 / 1.1$ \\
-2 & $0.68 / 0.4$ & $0.65 / 0.5$ & $0.55 / 0.9$ \\
-1 & $0.73 / 0.3$ & $0.71 / 0.4$ & $0.55 / 0.7$ \\
0 & $0.72 / 0.2$ & $0.56 / 0.3$ & $0.29 / 0.8$ \\
1 & $0.73 / 0.3$ & $0.35 / 0.4$ & $0.29 / 0.9$ \\
2 & $0.55 / 0.5$ & $0.24 / 0.4$ & $0.18 / 1.1$ \\
3 & $0.22 / 0.4$ & $0.21 / 0.3$ & $0.04 / 1.2$ \\
4 & $0.13 / 0.3$ & $0.19 / 0.3$ & $0.00 / 1.1$ \\
\hline
\end{tabular}

differences as are the divergence terms on the right of (4.11). The best results are obtained from

$$
T=D_{l}+D_{t}+B
$$

where the correlation of the left- and right-hand side climbs from 0.19 for $\tau=-4$ days to $\sim 0.7$ for $-2 \leq \tau \leq$ 1 day. Obviously, much of the observed tendency of the vorticity is explained by the advection terms and the $\beta$ term. In particular, the correlation is high for $\tau=-2$ days so that the vorticity equation captures the observed rapid increase of the perturbation amplitudes from $\tau=-2$ days until $\tau=0$. The pattern correlation coefficient $K$ does not reflect systematic amplitude errors. A gross measure of this error is given by the ratio $\sigma_{T} / \sigma_{R}$ of the standard deviations that should equal unity for a perfect model. It is seen from Table 1 that this ratio is $0.2-0.5$ for (4.12) so that the right-hand side of (4.11) overestimates the magnitude of the changes. Addition of the orographic term leads to a surprising deterioration for $0 \leq \tau \leq 2$ days. The importance of the turbulence term $D_{t}$ is underpinned by the relatively good success of the equation

$$
T=D_{t},
$$

where, in particular, $\sigma_{T} \approx \sigma_{R}$. Nevertheless, the correlations from (4.13) are always smaller than those obtained from (4.11) and (4.12).

Flux divergences on the right-hand side of (4.11), (4.12), and (4.13) are evaluated using centered differences, a procedure that involves substantial errors in such regions. This may explain the large values of $\sigma_{r}$. We can reduce these numerical problems by looking at selected regions and by simply summing the terms of (4.11) over the respective areas. In particular, it is found for a leeside region $\left(17^{\circ} \mathrm{W} \leq \lambda \leq 39^{\circ} \mathrm{W} ; 60^{\circ} \mathrm{N} \leq \varphi \leq\right.$ $77^{\circ} \mathrm{N}$ ) that there is no systematic overestimation by the thus integrated right-hand side of (4.11). Moreover, the incorporation of the orographic term is beneficial in this case in contrast to what has been concluded on the basis of Table 1. This discrepancy is obviously caused by finite difference errors.

Altogether we find that the vorticity Eq. (4.11) captures the evolution of the covariance fields reasonably well so that it makes sense to assess the contribution of its individual terms. The $\beta$ term $B$ reflects, of course, the vertical mean meridional wind component. It retards the eastward motion of the pressure systems but is certainly not responsible for the increase of the amplitudes observed for $-2 \leq \tau \leq 0$ days. This increase as well as the following decay must be caused by the divergence of the total flux $\gamma$ of relative vorticity. This term is not a source term but redistributes vorticity. Thus, any increase of $C(P, \zeta \mid \tau)$ at one spot must be associated with corresponding decreases elsewhere. The total flux $\gamma$ is displayed in Fig. 11. At $\tau=-2$ days, (Fig. 10a) there are large fluxes of vorticity out of the Greenland domain toward the south. These fluxes cause a growth of anticyclonic vorticity in the Greenland area and of cyclonic vorticity in the south. Two days later, (Fig. 10b) there is strong convergence of the fluxes above the tip and outflow from the Iceland and eastern Greenland domains favoring the growth of cyclonic vorticity near the tip and the eastward motion of the high (see Fig. 6). Further displacement to the east is documented in Fig. 10c.

An inspection of the contributions of the fluxes $\gamma_{i}$ to Fig. 10 shows that none of these fluxes can be neglected although $\gamma_{2}$ is somewhat smaller than the others. All fluxes are displayed in Fig. 11 for the lag $\tau=-2$ days. Clearly, $\boldsymbol{\gamma}_{1}$ must be essentially parallel or antiparallel to the vertical mean of the climatological flow so that transports toward the south must be expected above Greenland, where the vorticity covariance tends to be negative and the mean winds are southerly (Fig. 11a). These fluxes contribute to an intensification of anticyclonic vorticity in the northern part of the domain and to a buildup of cyclonic vorticity in the south. The flux $\gamma_{2}$ is by and large oriented along or opposite to the vertical mean wind correlation. The background vorticity $\bar{\zeta}$ is positive in the trough in the western part of our domain so that Fig. 11b shows northward transports of vorticity on the western side of the high above Greenland (see also Fig. 6). This term contributes to a strengthening of the high near Iceland by exporting cyclonic vorticity from this region. The turbulent fluxes (Fig. 11c) are directed southward in the eastern part of the domain, thus strengthening the high in the lee of Greenland and the cyclonic circulation in the south. The turbulent fluxes appear to converge in the low 

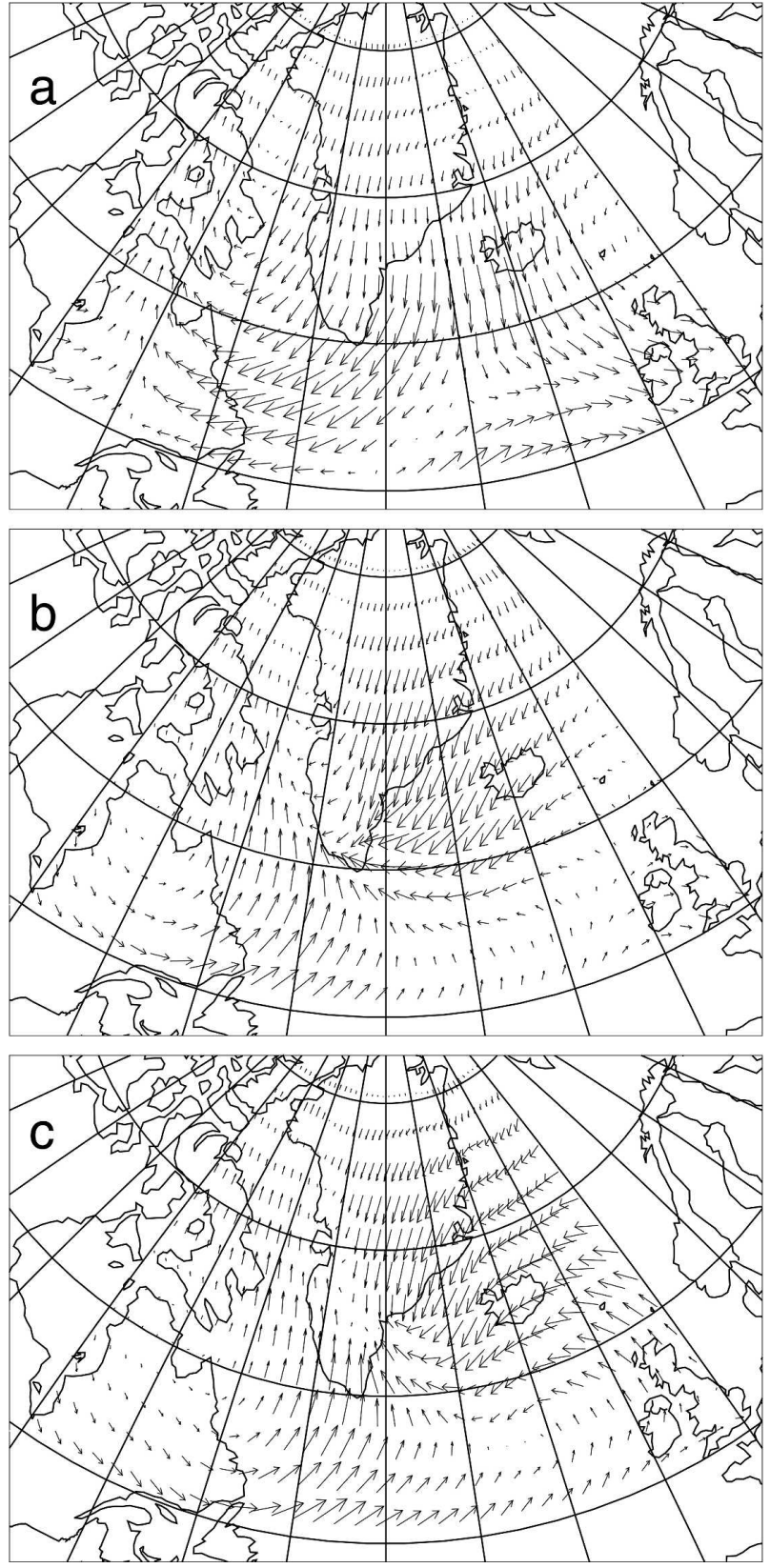

FIG. 10. Vertical mean covariance $\gamma$ [see (4.5)] of the Greenland mountain torque with the vorticity flux at (a) $\tau=-2$ days (max vector length $1.3 \times 10^{-4} \mathrm{~m} \mathrm{~s}^{-2}$ ), (b) $\tau=0$ days ( $\max$ vector length $2.9 \times 10^{-4} \mathrm{~m} \mathrm{~s}^{-2}$ ), and (c) $\tau=1$ day (max vector length $1.7 \times 10^{-4}$ $\mathrm{m} \mathrm{s}^{-2}$ ).

pressure region above Quebec. It is clear from these figures that the turbulent fluxes dominate east of Greenland and in the southwestern cyclone region while $\gamma_{1}$ is important above Greenland.

The changes of the fluxes $\gamma_{1}$ and $\gamma_{2}$ with increasing lag reflect the strengthening and eastward motion of the pressure systems but $\gamma_{1}$ remains similar to Fig. 11a for all $\tau$. The same is true with respect to $\gamma_{2}$. On the other hand, $\gamma_{3}$ is quite variable. The southward fluxes in Fig. 11c are replaced by a flux convergence at the southern tip at $\tau=0$ (Fig. 11d) so that turbulence contributes to the growth of cyclonic vorticity there. One day later, $\gamma_{3}$ is again rather different from the earlier patterns with strong northward fluxes east of Iceland and above Greenland (Fig. 11d). The corresponding evaluation of flux divergences shows that $D_{t}$ favors cyclonic growth over Quebec and anticyclonic one above and east of the massif at $\tau=-2$ days, that is, $D_{t}$ clearly supports the growth of the centers. At $\tau=0$, the Greenland area is dominated by cyclonic values of $D_{t}$, the east is anticyclonic so that again $D_{t}$ supports the development. There is a center of extremely high, but presumably spurious, cyclonic tendencies above the tip of Greenland (see Fig. 11d). At $\tau=1$ day, anticyclonic tendencies prevail in the south, cyclonic ones in the north.

The results presented so far suggest strongly that the triple terms are key factors in the intensification process. A firm proof of this suggestion is obtained by integrating the linear vorticity equation

$$
\begin{aligned}
\frac{\partial}{\partial t} C(P, \zeta \mid \tau)= & -\overline{\mathbf{v}} \cdot \boldsymbol{\nabla}(P, \zeta \mid \tau)-C(P, \mathbf{v} \mid \tau) \cdot \nabla(\bar{\zeta}+f) \\
& -\frac{f_{\alpha} \alpha C\left(P, \mathbf{v}_{s} \cdot \nabla h \mid \tau\right)}{H}
\end{aligned}
$$

where

$$
\begin{aligned}
& C(P, \mathbf{v} \mid \tau)=C(P, \mathbf{k} \times \nabla \psi \mid \tau) \quad \text { and } \\
& C(P, \zeta \mid \tau)=C\left(P, \nabla^{2} \psi \mid \tau\right),
\end{aligned}
$$

with streamfunction $\psi$ and an orographic fudge factor $0<\alpha \leq 1$. The integration starts at $\tau=-2$ days, that is when the intensification process begins. The initial vorticity covariance in (4.14) is the observed one. Boundary values are updated according to observations. Inversion of the Laplacian yields the streamfunction. Advection in (4.14) is formulated in terms of an upstream scheme but with a short time step $(D t=30$ min.) to minimize the damping effect of this scheme (e.g., Durran 1999). This initial vorticity distribution (not shown) is dominated by anticyclonic vorticity with a center of $-7 \times 10^{6} \mathrm{~s}^{-1}$ above Greenland. Cyclonic domains are seen above Canada and the southern part of the Atlantic. The linear model predicts for $\tau=0$, a northeastward shift and weakening of the anticyclonic centers and a northward extension of the cyclonic domains but without intensification (Fig. 12a). This process continues until $\tau=2$ days when the cyclonic domain occupies almost the complete analysis domain. 

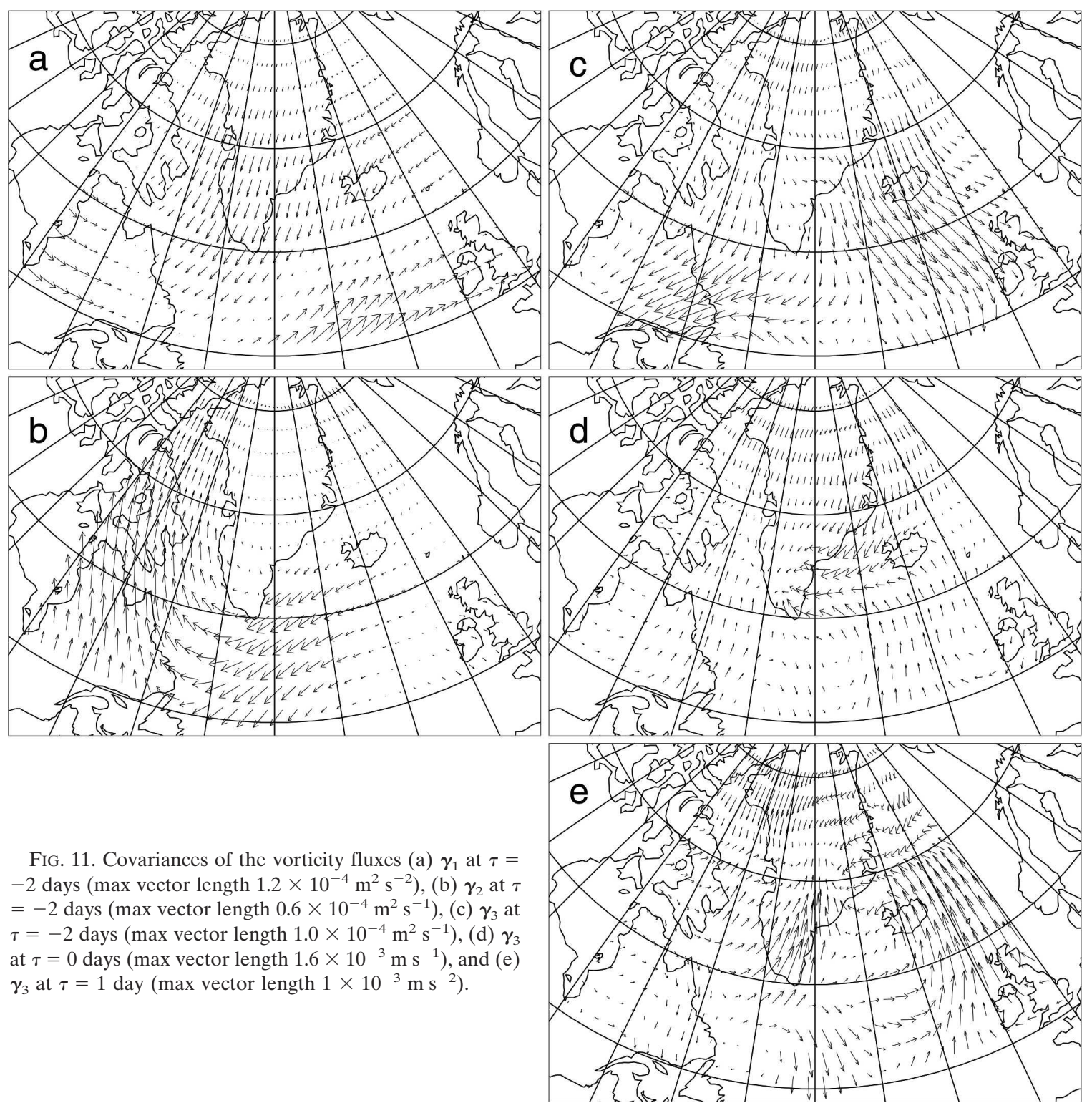

FIG. 11. Covariances of the vorticity fluxes (a) $\gamma_{1}$ at $\tau=$ -2 days (max vector length $1.2 \times 10^{-4} \mathrm{~m}^{2} \mathrm{~s}^{-2}$ ), (b) $\gamma_{2}$ at $\tau$ $=-2$ days $\left(\max\right.$ vector length $\left.0.6 \times 10^{-4} \mathrm{~m}^{2} \mathrm{~s}^{-1}\right)$, (c) $\gamma_{3}$ at $\tau=-2$ days (max vector length $1.0 \times 10^{-4} \mathrm{~m}^{2} \mathrm{~s}^{-1}$ ), (d) $\gamma_{3}$ at $\tau=0$ days (max vector length $1.6 \times 10^{-3} \mathrm{~m} \mathrm{~s}^{-1}$ ), and (e) $\gamma_{3}$ at $\tau=1$ day (max vector length $1 \times 10^{-3} \mathrm{~m} \mathrm{~s}^{-2}$ ).

The linear prediction captures the overall distribution of positive and negative vorticities reasonably well, but fails completely with respect to the observed leeside anticyclonic center as seen in Fig. 12b. Enhancement of $\alpha$ from 0.25 as in Fig. 12a to $\alpha=1$ leads to the generation of a spurious center of cyclonic vorticity in the lee. The covariance enstrophy of this run increases slightly from $\tau=-2$ days until $\tau=0$ (Fig. 9). Obviously, the linear theory misses the intensification process almost completely. This demonstrates clearly that the triple terms are crucial.

At the end, a word on statistical significance may be in order. It is customary to estimate the significance of covariance fields pointwise. Given the large number of observations all but the weakest signals turn out to be highly significant. Of course (e.g., von Storch and Zwiers 1999), this procedure is not satisfactory because there are so many points in one field and because grid points are not independent. However, the fields shown here are so smooth and are interpretable in terms of the vorticity equation that there is no pressing need to use elaborate methods based on data reduction and Monte Carlo tests.

\section{Discussion and conclusions}

The rapid increase of the amplitude of the covariances of mountain torque and flow variables for $\tau \rightarrow 0$ 


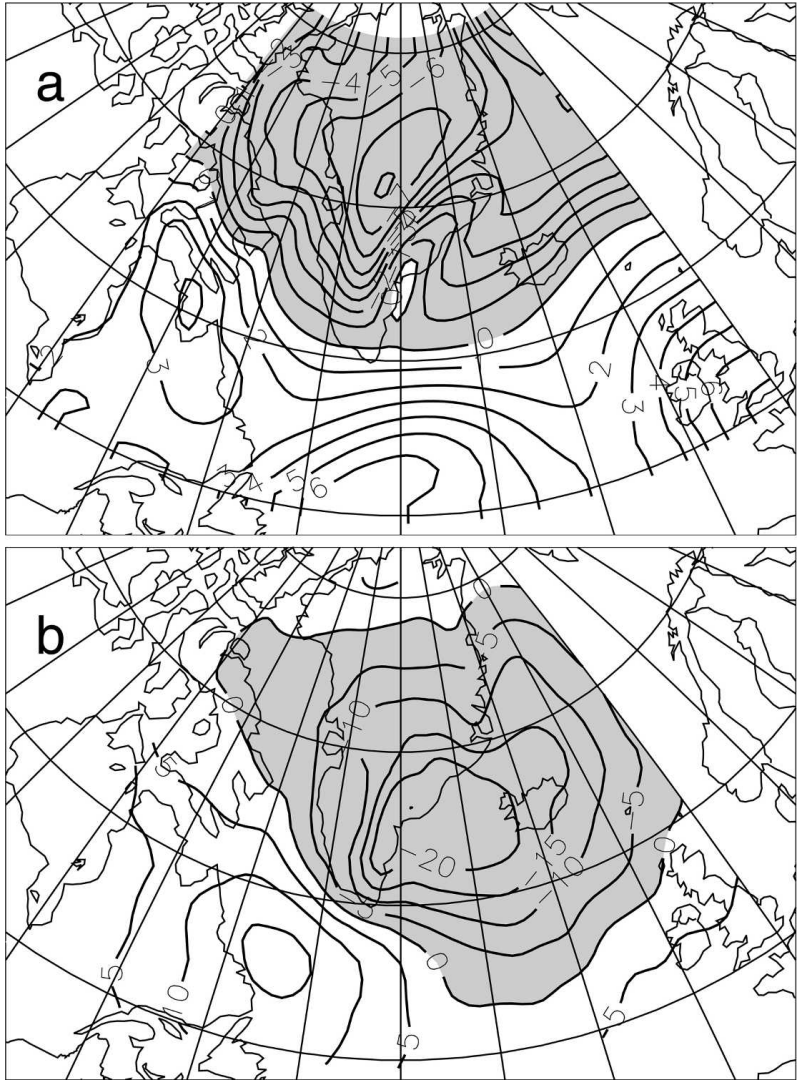

FIG. 12. Vertical mean vorticity covariance $C(P, \zeta \mid \tau)\left(10^{-6} \mathrm{~s}^{-1}\right)$ at $\tau=0$ days as (a) predicted by the linear vorticity equation and (b) observed with $\alpha=0.25$.

in the CDV model is mainly caused by the stochastic forcing but the triple covariances are also important. Our analysis suggests that the processes behind these terms are akin to those encountered in the unforced version of this model. They reflect the energy transfer between mean flow and wave via the mountain.

The analysis of the Greenland case revealed that positive torque events involve the growth of deep high pressure systems above and to the east of Greenland while a low is moving southeastward off the tip of Greenland. A fast increase of the covariances of all fields with the mountain torque is found in this case as well for $\tau \rightarrow 0$. There is no support for Scorer's (1988) suggestion that the Icelandic low is a leeside low. The angular momentum in the analysis domain decreases during a positive torque event because of the boundary fluxes.

The two-dimensional vorticity equation has been used as a diagnostic tool in vertically integrated form. It has been shown that this equation is useful even near and above the steep topography of Greenland. Although the linear terms are clearly important in the
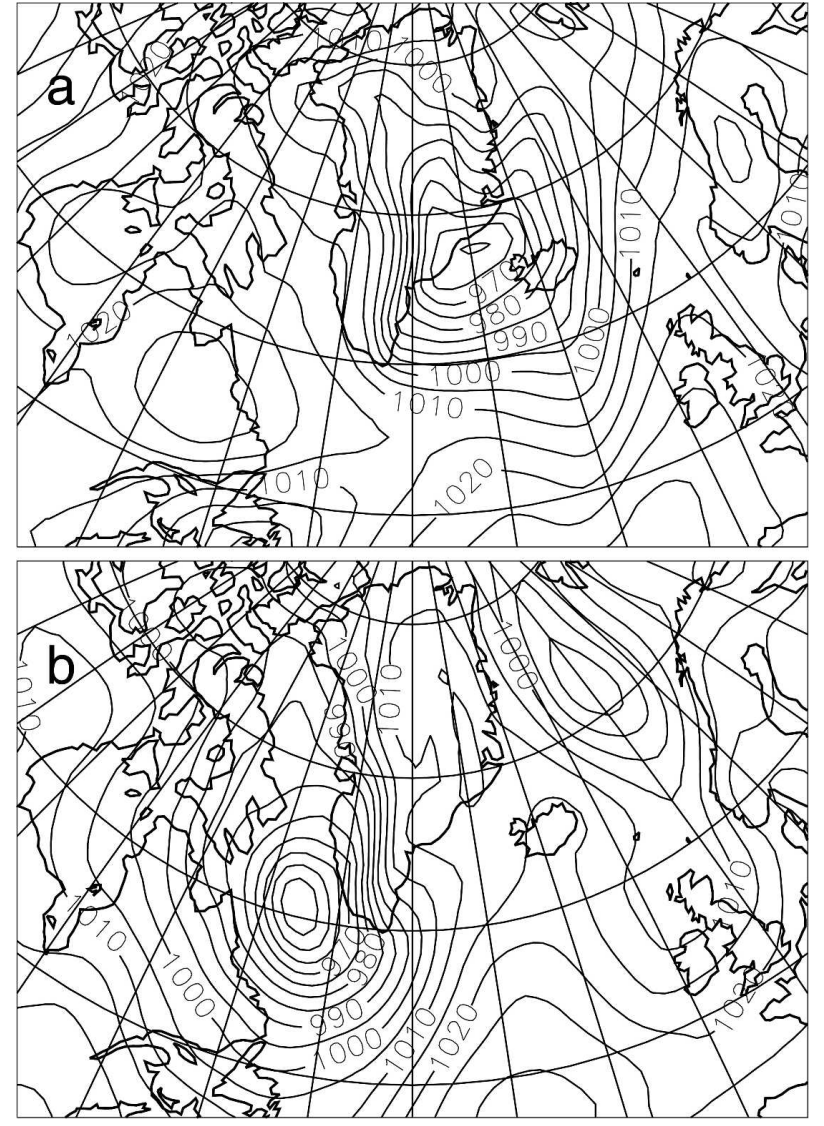

FIG. 13. Surface pressure maps (hPa) for (a) 9 Jan 1984 with $P=-20.2 \mathrm{Ha}$ and (b) 24 Nov 1962 with $P=20.3 \mathrm{Ha}$.

dynamics of the torque events, the triple terms are indispensable both in the cyclonic region in the south and near and above Greenland. This statement is strongly supported by the results obtained from the linear vorticity equation. The failure of the linear calculations suggests that an approach along the lines of WS98 would not be successful in the Greenland case. The perturbations receive little energy from the mean flow.

The triple terms change rapidly with lag and are difficult to interpret. In the broadest sense they represent the transfer of energy from nonstationary motion at larger scales to the relatively small centers of action near Greenland. This transfer is almost certainly supported by the orography. It adds to the problems of interpretation that the covariances as calculated are linear in the sense that events with negative torque are obtained from those with $P>0$ by a simple switch of the sign, a feature which may not hold in reality. This point can be clarified at least partially by looking at pairs of individual cases with opposite values of the torque. The surface pressure distributions for such a pair are shown in Fig. 13. For $P=20.3$ Ha (Fig. 13b), 

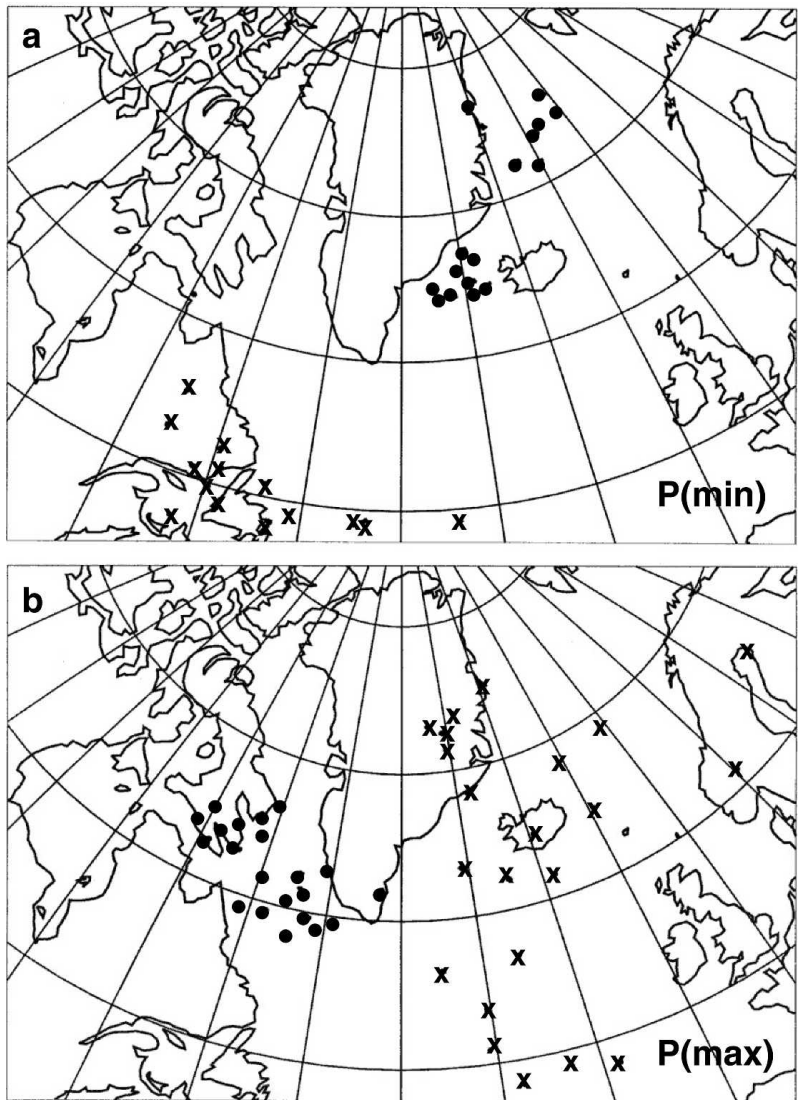

FIG. 14. Positions of the centers of lows (dots) and highs (crosses) near Greenland for days with (a) $P \leq-20 \mathrm{Ha}$ and (b) $P$ $\geq 20 \mathrm{Ha}$.

there is a deep cyclone west of the tip and high pressure ridge extending from the south toward Iceland. The case with $P=-20.2 \mathrm{Ha}$ has a high above Canada and a low in the lee of Greenland (Fig. 13a). Both cases are similar to Fig. 6e. It is clear, however, from both figures that the lows are deeper than the highs corresponding with generally accepted synoptic experience. Hoskins and Hodges (2002) show that more cyclones than anticyclones pass near Greenland and that the maximum of anticyclonic track density is located south of that of cyclones. An inspection of all cases with strong torques $|P| \geq 20$ Ha shows that the low pressure centers (Fig. 14) are found in a distinct domain while those of the highs are fairly widespread. For $P>20 \mathrm{Ha}$ the locations of the highs are spread all over the Atlantic while those of the lows are concentrated near the tip of Greenland (Fig. 14b). The lows are aligned along the Greenland coast for $P<-20 \mathrm{Ha}$ (Fig. 14a). The statistical procedures used above include all cases with proper weight. It is for this reason that the low in Fig. 6e is located in between the positions of the lows west of Greenland in Fig. 14b and the highs above Canada in Fig. 14a. The same way, the turbulent vorticity fluxes in Fig. 11d can be understood only if these features are taken into account. The situation found here is similar to but slightly more complicated than that in climatological budget studies where time mean fields interact with turbulent transports. In our case, the basic variables are covariances interacting with triple terms and the interaction evolves with lag. Further progress has presumably to come from the investigations of the vorticity transport in individual cases.

The location of Greenland poleward of a storm track is fairly unique. It will have to be found out by further work if the main results of this paper are also valid at other mountain ranges.

Acknowledgments. We are grateful to Klaus Weickmann and an anonymous referee for perceptive and helpful comments.

\section{REFERENCES}

Bougeault, P., and Coauthors, 1993: The atmospheric momentum budget over a major mountain range: First results from the PYREX field experiment. Ann. Geophys., 11, 395-418.

Charney, J., and J. DeVore, 1979: Multiple flow equilibria and blocking. J. Atmos. Sci., 36, 1205-1216.

Crommelin, D., and A. Majda, 2004: Strategies for model reduction: Comparing different optional bases. J. Atmos. Sci., 61, 2206-2217.

Czarnetzki, A., 1997: Regional mountain torque estimate over the Rocky Mountains in lee cyclones. J. Atmos. Sci., 54, 1986-1987.

Doyle, J., and M. Shapiro, 1999: Flow response to large-scale topography: The Greenland tip jet. Tellus, 51A, 728-748.

Durran, D., 1999: Numerical Methods for Wave Equations in Geophysical Fluid Dynamics. Springer, 463 pp.

Egger, J., 1981: Stochastically driven large-scale circulations with multiple equilibria. J. Atmos. Sci., 38, 2606-2618.

— , and W. Metz, 1981: On the mountain torque in barotropic planetary flow. Quart. J. Roy. Meteor. Soc., 107, 299-312.

— ability of the atmosphere. J. Atmos. Sci., 40, 1073-1085.

Hoskins, B., and K. Hodges, 2002: New perspectives on the Northern Hemisphere winter storm tracks. J. Atmos. Sci., 59, 10411061.

Jin, F.-F., and M. Ghil, 1990: Intraseasonal oscillations in the extratropics: Bifurcations and topographic instability. J. Atmos. Sci., 47, 3007-3022.

Kristjánsson, J., and H. McInnes, 1999: The impact of Greenland on cyclone evolution in the North Atlantic. Quart. J. Roy. Meteor. Soc., 125, 2819-2834.

Lott, F., A. Robertson, and M. Ghil, 2004: Mountain torques and Northern Hemisphere low-frequency variability. Part II: Regional aspects. J. Atmos. Sci., 61, 1272-1282.

Oort, A., 1989: Angular momentum cycle in the atmosphereocean-solid earth system. Bull. Amer. Meteor. Soc., 70, 12311242.

Putnins, P., 1970: The climate of Greenland. Climates of the Polar Regions, S. Orvig, Ed., World Survey of Climatology, Vol. 14, Elsevier, 1-128. 
Schwierz, C., 2001: Interactions of Greenland-scale orography and extra-tropical synoptic-scale flow. Ph.D. dissertation, Swiss Federal Institute of Technology, 155 pp. [Available upon request from the author: schwierz@atmos.umnw.ethz.ch.]

Scorer, R., 1988: Sunny Greenland. Quart. J. Roy. Meteor. Soc., 114, 3-29.

Simmons, A., and J. Gibson, 2000: The ERA-40 Project Plan: ERA-40 Project Rep. Series 1, ECMWF, Shinfield Park, Reading, United Kingdom, 63 pp.

Tung, K., and A. Rosenthal, 1985: Theories of multiple equilibria, a critical reexamination. Part I: Barotropic models. J. Atmos. Sci., 42, 2806-2819.

von Storch, H., and F. Zwiers, 1999: Statistical Analysis in Climate Research. Cambridge University Press, 484 pp.
Walden, H., 1959: Statistisch-synoptische Untersuchung über das Verhalten von Tiefdruckgebieten im Bereich von Grönland (Statistic-synoptic investigation on the behavior of lows within the range of Greenland). Deutscher Wetterdienst, 70 pp.

Wallace, J., G. Lim, and M. Blackmon, 1988: Relationship between cyclone tracks, anticyclone tracks and baroclinic waveguides. J. Atmos. Sci., 45, 439-462.

Weickmann, K., 2003: Mountains, the global frictional torque and the circulation over the Pacific-North American region. Mon. Wea. Rev., 131, 2608-2622.

Whitaker, J., and P. Sardeshmukh, 1998: A linear theory of extratropical synoptic eddy statistics. J. Atmos. Sci., 55, 237258 . 